\title{
Análise de sobrevida em pacientes com câncer de pulmão tratados no Sistema Único de Saúde no Brasil entre 2002 e 2003
}

\author{
Survival analysis in patients with lung cancer \\ treated in Unified Health System in Brazil between 2002 and 2003
}

\author{
Carlos Philipe Barbosa Polato ${ }^{1}$, Gisele Macedo da Silva Bonfante², Eli lola Gurgel Andrade², \\ Francisco de Assis Acurcio ${ }^{4}$, Carla Jorge Machado ${ }^{5}$, Mariângela Leal Cherchiglia ${ }^{6}$
}

\begin{abstract}
Resumo
Introdução: O câncer representa uma crise de saúde pública mundial. No Brasil, são 519 mil casos novos de câncer em 2012-2013 e já é a segunda causa de morte. O câncer de pulmão é um dos tipos mais comuns. O objetivo deste estudo foi realizar a análise de sobrevida em pacientes com câncer de pulmão tratados no Sistema Único de Saúde. Método: Por meio de pareamento determinístico-probabilístico, foram reunidos dados de tratamento oncológico prestados pelo Sistema Único de Saúde num mesmo cadastro, sendo criada a Base Nacional em Oncologia. Foi realizada uma análise descritiva e uma análise de sobrevida no software SPSS versão $20^{\circledR}$, sendo adotado um nível de significância de $5 \%$. Resultados: Foram analisados 11.157 pacientes. A maioria deles $(88,8 \%)$ foi diagnosticada e tratada com doença avançada. A sobrevida para o câncer de pulmão inferior a 1 ano foi de $45 \%$, e a sobrevida para o câncer de pulmão superior a 5 anos foi de $16 \%$. Discussão: A maioria dos pacientes foi diagnosticada em estádios avançados e o óbito por câncer de pulmão foi o desfecho mais comum. Conclusão: O câncer de pulmão é uma doença grave, de difícil diagnóstico e de difícil tratamento, sendo a prevenção a melhor conduta.
\end{abstract}

Palavras-chave: análise de sobrevida; neoplasias pulmonares; Brasil.

\begin{abstract}
Introduction: Cancer is a worldwide public health crisis. In Brazil there are 519,000 new cases of cancer in 2012-2013 and is the second cause of death. Lung cancer is one of the most common types. The aim of this study was to analyze the survival of patients with lung cancer treated in the Unified Health System. Method: Through pairing deterministic-probabilistic data were gathered oncology treatment provided by the Unified Health System in the same register, being created National Basis in Oncology. We performed a descriptive analysis and a survival analysis with SPSS ${ }^{\circledR}$ version 20 , adopting a significance level of $5 \%$. Results: We analyzed 11,157 patients. Most of them (88.8\%) were diagnosed and treated with advanced disease. The survival
\end{abstract}

Trabalho realizado no Programa de Pós-Graduação em Saúde Pública (PPGSP) da Faculdade de Medicina da Universidade Federal de Minas Gerais (UFMG) Belo Horizonte (MG), Brasil.

${ }^{1}$ Mestre em Saúde Pública pela Faculdade de Medicina da UFMG; Médico anestesiologista do Hospital Municipal Odilon Behrens (HOB) - Belo Horizonte (MG) - Brasil. 2Doutoranda em Epidemiologia pela Faculdade de Medicina da UFMG; Cirurgiã-Dentista do Exército Brasileiro (EB) - Belo Horizonte (MG) - Brasil.

${ }^{3}$ Doutora em Demografia pela Faculdade de Economia da UFMG; Professora associada da Faculdade de Medicina da UFMG - Belo Horizonte (MG) - Brasil.

4Pós-Doutor em Economia da Saúde pela Universitat Pompeu Fabra (Espanha); Professor titular do Departamento de Farmácia Social da Faculdade de Farmácia da UFMG - Belo Horizonte (MG) - Brasil.

${ }^{5}$ Pós-Doutora em Epidemiologia pela Faculdade de Medicina da UFMG; Professora associada da Faculdade de Medicina da UFMG - Belo Horizonte (MG) - Brasil. ${ }^{6}$ Pós-Doutora em Saúde Pública pela Universidade Nova de Lisboa (Portugal); Professora associada da Faculdade de Medicina da UFMG - Belo Horizonte (MG) - Brasil. Endereço para correspondência: Carlos Philipe Barbosa Polato - Grupo de Pesquisa em Economia da Saúde (GPES) - Faculdade de Medicina da Universidade Federal de Minas Gerais - Avenida Alfredo Balena, 190, $7^{\circ}$ andar - CEP: 30190-170 - Belo Horizonte (MG), Brasil - E-mail: philipepolato@hotmail.com.

Fonte de financiamento: nenhuma.

Conflito de interesse: nada a declarar. 
rate for lung cancer in less than 1 year was $45 \%$ and survival for lung cancer over 5 years was $16 \%$. Discussion: The majority of patients were diagnosed in advanced stages and death from lung cancer was the most common outcome. Conclusion: Lung cancer is a serious disease, difficult to diagnose and difficult to treat, being prevention the best approach.

Keywords: survival analysis; lung neoplasms; Brazil.

\section{INTRODUÇÃO}

Até recentemente, o câncer foi considerado uma doença de países ocidentais, dos países industrializados. Hoje a situação mudou radicalmente, com a maioria do ônus global do câncer agora encontrada em países em desenvolvimento, que muitas vezes têm um orçamento de saúde limitado e um elevado nível residual de doenças transmissíveis e onde as instalações de tratamento de câncer e as terapias não são universalmente disponíveis ${ }^{1-3}$.

O rápido aumento da carga de câncer representa uma verdadeira crise de saúde pública mundial. Uma questão importante para muitos países, mesmo entre os países desenvolvidos, será como encontrar recursos suficientes para tratar de forma eficaz e oferecer cuidados de suporte para o grande número de pacientes que serão diagnosticados nos próximos anos ${ }^{1-3}$.

No Brasil, as estimativas para o ano de 2012 serão válidas também para o ano de 2013 e apontam a ocorrência de aproximadamente 519 mil casos novos de câncer, incluindo os casos de pele não melanoma, o que representa a segunda causa de morte no país, reforçando a magnitude do problema do câncer aqui ${ }^{1-3}$.

O câncer afeta crianças, adultos e idosos; homens e mulheres; sem qualquer discriminação de classe social ou etnia. Representa uma carga enorme para os pacientes, suas famílias e para toda a sociedade ${ }^{1-3}$.

Entre os tipos de câncer, o câncer de pulmão é um dos mais comuns no mundo. No Brasil, apresenta-se como o segundo câncer mais comum em homens e o quinto em mulheres, excetuando-se os cânceres de pele não melanoma. Entre os tipos de câncer de pulmão dois são mais frequentes, o Carcinoma de Pulmão de Células não Pequenas (cerca de 65 a 75\% do total) e o Carcinoma de Pulmão de Células Pequenas (cerca de 20 a $25 \%$ do total) ${ }^{1,2,4}$.

O hábito de consumo de tabaco é o mais importante fator de risco para o desenvolvimento do câncer de pulmão. Em geral, as taxas de incidência em um determinado país refletem seu consumo de cigarros. No fim do século XX, o câncer de pulmão se tornou uma das principais causas de morte prevenível. De fato, estima-se que 80 a 90\% da incidência de câncer de pulmão sejam atribuídos ao fumo. Comparados com os não fumantes, os tabagistas têm cerca de 20 a 30 vezes o risco dos não fumantes de desenvolverem câncer de pulmão ${ }^{2}$.
Devido à dificuldade para o diagnóstico precoce, a maioria apresenta estádios avançados no momento do diagnóstico. Somente um terço dos pacientes submete-se à retirada total cirúrgica do tumor e, portanto, a maioria não é candidata a tratamento curativo. A maior parte dos casos necessita de tratamentos paliativos: quimioterapia, radioterapia ou ambos. Em decorrência disso, o câncer de pulmão permanece como uma doença altamente letal ${ }^{5}$.

O grupo com sobrevida superior a cinco anos apresenta estádios mais precoces, com doenças mais localizadas e capacidade funcional melhor, o que permite que os pacientes consigam as condições adequadas para a cirurgia, ou seja, que o tumor seja ressecável e, por conseguinte, que sua sobrevida seja maior do que cinco anos após o diagnóstico ${ }^{5}$.

Quanto às taxas de mortalidade por câncer de pulmão variam consideravelmente entre os países. Uma das razões para isso é que os valores de sobrevida citados na literatura são baseados em dados que variam amplamente em sua coleta e análise estatística e esta informação nem sempre é de domínio público. Publicações internacionais de sobrevida em 5 anos para pacientes com câncer de pulmão variam de 5 a 16\%. Dados dos Estados Unidos sugerem uma taxa de sobrevida global em 5 anos de até 16\%, embora esse número inclua apenas $1 / 4$ da população em geral e exclua pacientes sem confirmação histológica. Muitos países europeus relatam taxas de mortalidade mais elevadas, embora na maioria das vezes os dados incluam pacientes sem histologia comprovada ${ }^{6}$.

Devido às altas taxas de mortalidade do câncer de pulmão tornam-se imperativas estratégias de prevenção e controle do câncer, assim como são importantíssimas as informações de qualidade sobre a distribuição de incidência e mortalidade para ampliar a compreensão da doença e de seus determinantes. Um sistema de vigilância estruturado fornece informações sobre a magnitude e o impacto do câncer, bem como sobre a efetividade dos programas de controle. Os registros de câncer de base populacional e hospitalar e as informações sobre mortalidade são parte desse sistema de vigilância ${ }^{2}$.

O objetivo deste estudo foi realizar a análise de sobrevida inferior a 1 ano e superior a 5 anos em pacientes maiores de 18 anos submetidos à cirurgia, à radioterapia e/ou à quimioterapia para o tratamento do câncer de pulmão no SUS no período de $1^{\circ}$ de janeiro 2002 a 31 de dezembro 2003. 


\section{MÉTODO}

Trata-se de um estudo do tipo observacional, prospectivo não concorrente, que utilizou, como fonte de dados, a Base Nacional em Oncologia, a Base Onco. Essa base foi desenvolvida por meio da técnica de pareamento determinístico-probabilístico utilizando os bancos de dados do Subsistema de Autorização para Procedimentos de Alto Custo/Complexidade (APAC) do Sistema de Informações Ambulatoriais do SUS (SIA/SUS), de Autorização de Internação Hospitalar (AIH) do Sistema de Informações Hospitalares do SUS (SIH/SUS) e do Sistema de Informações sobre Mortalidade (SIM), com o objetivo de habilitar o seguimento de uma coorte histórica que envolve pacientes submetidos à radioterapia e/ou à quimioterapia com ou sem cirurgia associada, para o tratamento do câncer de pulmão no SUS no período de $1^{\circ}$ de janeiro de 2002 a 31 de dezembro de 2003.

Conforme prevê a Resolução no 196/96 do Conselho Nacional de Saúde (CNS), a consulta à base de dados foi realizada após autorização e assinatura de um Termo de Compromisso com o Grupo de Pesquisa em Economia da Saúde (GPES), que já havia submetido o estudo "Avaliação econômico epidemiológica do tratamento oncológico no Sistema Único de Saúde", no qual foi desenvolvida a Base Nacional em Oncologia, a Base Onco, à apreciação do Comitê de Ética em Pesquisa da Universidade Federal de Minas Gerais (COEP - UFMG). Esse estudo recebeu parecer favorável em 29 de abril de 2009 sob o número ETIC 072/09. Ressaltamos que não houve contato direto com os pacientes e não houve ônus para a instituição onde se realizou a pesquisa. Os custos referentes ao material de consumo foram arcados pelos pesquisadores.

A primeira fonte de dados a ser utilizada foram informações disponibilizadas no Subsistema APAC do SIA/SUS, selecionando especificamente os procedimentos de relevância para o estudo, procedimentos de radioterapia (código 28 da APAC) e quimioterapia (código 29 da APAC), nos pacientes com câncer de pulmão (código C34 da CID 10), por meio do pareamento determinístico-probabilístico, o que permitiu a geração de um cadastro único de pacientes que realizaram tratamento radioterápico e/ou quimioterápico no SUS no período de 2000 a 2006.

A seguir os dados relativos às cirurgias oncológicas, disponibilizados nas bases de dados de AIH do SIH/SUS, nos pacientes com câncer de pulmão (código C34 da CID 10), por meio do pareamento determinístico-probabilístico permitiram a geração de um cadastro único de pacientes que realizaram tratamento cirúrgico no SUS no período de 2000 a 2005.

Assim, por intermédio do pareamento determinístico-probabilístico, em um mesmo cadastro foram reunidos todos os dados de tratamento oncológico prestados pelo SUS no período para um mesmo paciente (cirurgia, radioterapia e/ou quimioterapia), sendo então criada a Base Nacional em Oncologia, a Base Onco.

Os dados relativos à ocorrência de óbitos foram confirmados por meio de pareamento determinístico-probabilístico entre a Base Onco e o SIM, disponibilizado com dados completos para o período de 2003 a 2008.

As bases de dados do Subsistema APAC do SIA/SUS e as bases de dados de AIH do SIH/SUS foram obtidas com o Departamento de Informática do SUS (DATASUS) e com a Secretaria de Assistência à Saúde do Ministério da Saúde (SAS/MS); e as bases de dados do SIM foram obtidas com a Secretaria de Vigilância em Saúde do Ministério da Saúde (SVS/MS).

A utilização da técnica de pareamento determinístico-probabilístico para composição da Base Onco seguiu as mesmas estratégias utilizadas pela Base Nacional em Terapia Renal Substitutiva (TRS), desenvolvida pelo GPES anteriormente ${ }^{7}$.

Foram incluídos na presente investigação pacientes pertencentes à Base Onco, diagnosticados como câncer de pulmão e submetidos à radioterapia e/ou à quimioterapia com ou sem cirurgia associada, para o tratamento do câncer de pulmão no SUS no período de $1^{\circ}$ de janeiro de 2002 a 31 de dezembro de 2003 (código C34 da CID 10 no momento do diagnóstico). Os pacientes com idade inferior ou igual a 18 anos foram excluídos.

As variáveis estudadas foram sociodemográficas (sexo, idade ao diagnóstico, região de nascimento e região de residência) e clínicas (estadiamento no momento do diagnóstico, modalidade de tratamento (cirurgia, radioterapia e/ou quimioterapia), ocorrência de metástase, tempo entre diagnóstico e ocorrência de metástase, tempo entre diagnóstico e entrada em tratamento, ocorrência de outro câncer principal e resultado final).

Foi realizada uma análise descritiva por meio de distribuições de frequências, medidas de tendência central e de variabilidade para as características estudadas.

Para as análises de sobrevida global foram consideradas falhas aqueles pacientes cujos óbitos estiveram relacionados a qualquer causa. Foram censurados os pacientes que receberam alta ou que permaneceram vivos até o término do estudo. Os pacientes foram acompanhados a partir da data de seu diagnóstico até o término do estudo.

Para as análises de sobrevida específica por câncer de pulmão foram consideradas falhas aqueles pacientes cujos óbitos estiveram relacionados ao câncer de pulmão. Foram censurados os pacientes que receberam alta ou que permaneceram vivos até o término do estudo ou que foram a óbito por outras 
causas. Os pacientes foram acompanhados a partir da data de seu diagnóstico até o término do estudo.

As funções de sobrevida foram calculadas a partir do método de Kaplan-Meier, segundo as variáveis explicativas do estudo e o teste de Log-Rank foi empregado para compará-las. Para avaliação dos fatores associados à sobrevida, foi empregado o Modelo de Cox, computando-se os hazard-ratios com os respectivos IC95\%.

As análises foram realizadas no software SPSS versão $20^{\circledR}$, sendo adotado um nível de significância de $5 \%$.

\section{RESULTADOS}

Foram analisados 11.157 pacientes diagnosticados como câncer de pulmão e submetidos à radioterapia e/ou à quimioterapia com ou sem cirurgia associada, para o tratamento do câncer de pulmão no SUS no período de $1^{\circ}$ de janeiro de 2002 a 31 de dezembro de 2003. (Tabela 1). A maior parte desses pacientes $(n=7.605 ; 68,2 \%)$ era do sexo masculino. A idade variou de 19 a 100 anos, com média de 61,7 anos. Quase a totalidade desses pacientes era de brasileiros nato $(98,8 \%)$, nascidos no Sudeste $(47,9 \%)$, seguido do Sul $(26,4 \%)$, Nordeste $(16,3 \%)$, Centro-Oeste $(5,1 \%)$ e Norte $(3,1 \%)$. Além disso, a maioria desses pacientes residia à época no Sudeste $(49,5 \%)$ e no Sul $(27,6 \%)$, os demais residiam no Nordeste $(14,3 \%)$, no Centro-Oeste $(5,3 \%)$ ou no Norte $(3,2 \%)$.

A grande maioria dos pacientes $(88,8 \%)$ foi diagnosticada e tratada com doença avançada (estádios III e IV). Entre os 11.157 (100\%) pacientes, $4.128(37,0 \%)$ passaram por internações para a realização de algum tipo de procedimento, sendo que destes, 214 (1,9\%) passaram por internações para a realização de cirurgia para tratamento do câncer de pulmão; mais especificamente, 206 pacientes realizaram 1 cirurgia para tratamento do câncer de pulmão e 8 pacientes realizaram 2 cirurgias para tratamento do câncer de pulmão, totalizando 222 cirurgias para tratamento do câncer de pulmão; 7.077 $(63,4 \%)$ receberam radioterapia e 8.009 (71,8\%) receberam quimioterapia. Ocorreu metástase à distância em 5.576 pacientes $(50,0 \%)$. O tempo entre o diagnóstico e a ocorrência de metástase à distância variou de 0 a 47 meses, com média de 3,1 meses e mediana de 1,0 mês. O tempo entre o diagnóstico e a entrada em tratamento variou de 0 a 1.461 dias, com média de 73 dias e mediana de 31 dias. Quanto ao óbito, 74,4\% dos pacientes tiveram desfecho relacionado ao câncer de pulmão $(\mathrm{n}=8.304 ; 74,4 \%)$.

A análise de sobrevida global dos pacientes submetidos à radioterapia e/ou à quimioterapia com ou sem cirurgia associada, para o tratamento do câncer de pulmão no SUS no período de $1^{\circ}$ de janeiro de 2002 a 31 de dezembro de 2003 foi feita pelo método
Kaplan-Meier (Tabela 2). Dos 11.157 pacientes acompanhados em 83 meses, houve 10.065 óbitos por qualquer causa (90,2\%).

A análise de sobrevida específica dos pacientes submetidos à radioterapia e/ou à quimioterapia com ou sem cirurgia associada, para o tratamento do câncer de pulmão no SUS no período de $1^{\circ}$ de janeiro de 2002 a 31 de dezembro de 2003 foi feita pelo método Kaplan-Meier (Tabela 3). Entre os 11.157 pacientes acompanhados em 83 meses, houve 8.304 óbitos por câncer de pulmão $(74,4 \%)$. Houve uma maior sobrevida específica no sexo

Tabela 1. Características dos pacientes com câncer de pulmão tratados no SUS entre 2002 e 2003

\begin{tabular}{|c|c|}
\hline Características & n (\%) \\
\hline Total de pacientes & $11.157(100,0)$ \\
\hline \multicolumn{2}{|l|}{ Sexo } \\
\hline Masculino & $7.605(68,2)$ \\
\hline Feminino & $3.552(31,8)$ \\
\hline \multicolumn{2}{|l|}{ Idade ao diagnóstico (em anos) } \\
\hline$<50$ & $1.593(14,3)$ \\
\hline $50-59$ & $2.871(25,7)$ \\
\hline $60-69$ & $3.807(34,1)$ \\
\hline$\geq 70$ & $2.886(25,9)$ \\
\hline \multicolumn{2}{|l|}{ Região de nascimento } \\
\hline Sudeste & $5.346(47,9)$ \\
\hline Sul & $2.945(26,4)$ \\
\hline Centro-Oeste & $564(5,1)$ \\
\hline Norte & $350(3,1)$ \\
\hline Nordeste & $1.822(16,3)$ \\
\hline Naturalizados e estrangeiros & $130(1,2)$ \\
\hline \multicolumn{2}{|l|}{ Região de residência } \\
\hline Sudeste & $5.526(49,5)$ \\
\hline Sul & $3.084(27,6)$ \\
\hline Centro-Oeste & $592(5,3)$ \\
\hline Norte & $362(3,2)$ \\
\hline Nordeste & $1.593(14,3)$ \\
\hline \multicolumn{2}{|l|}{ Estadiamento no momento do diagnóstico } \\
\hline Doença precoce (estádios 0, I e II) & $1.254(11,2)$ \\
\hline Doença avançada (estádios III e IV) & $9.903(88,8)$ \\
\hline \multicolumn{2}{|l|}{ Modalidade de tratamento } \\
\hline Internações para algum tipo de procedimento & $4.128(37,0)$ \\
\hline $\begin{array}{l}\text { Internações para cirurgia para tratamento do } \\
\text { câncer de pulmão }\end{array}$ & $214(1,9)$ \\
\hline Radioterapia & $7.077(63,4)$ \\
\hline Quimioterapia & $8.009(71,8)$ \\
\hline \multicolumn{2}{|l|}{ Ocorrência de metástase } \\
\hline Não & $5.581(50,0)$ \\
\hline Sim & $5.576(50,0)$ \\
\hline \multicolumn{2}{|l|}{ Ocorrência de outro câncer principal } \\
\hline Não & $10.749(96,3)$ \\
\hline Sim & $408(3,7)$ \\
\hline \multicolumn{2}{|l|}{ Óbito relacionado ao câncer de pulmão } \\
\hline Não & $2.853(25,6)$ \\
\hline Sim & $8.304(74,4)$ \\
\hline
\end{tabular}

Fonte: Base Nacional em Oncologia 
feminino $(27,8 \%)$ e uma menor sobrevida específica no sexo masculino (24,5\%). Houve uma maior sobrevida específica na faixa etária $<50$ anos $(29,4 \%)$ e uma menor sobrevida específica na faixa etária $\geq 70$ anos $(24,4 \%)$. Houve uma maior sobrevida específica nos pacientes nascidos na região Norte $(43,7 \%)$ e uma menor sobrevida específica nos pacientes nascidos na região Sul (20,8\%), excetuando-se os pacientes naturalizados e estrangeiros $(19,2 \%)$. Houve uma maior sobrevida específica nos pacientes residentes na região Norte $(45,6 \%)$ e uma menor sobrevida específica nos pacientes residentes na região Sul (20,7\%). Houve uma maior sobrevida específica nos pacientes com doença precoce (estádios 0, I e II) (30,1\%) e uma menor sobrevida específica nos pacientes com doença avançada (estádios III e IV) (25,0\%). Houve uma maior sobrevida específica nos pacientes sem nenhuma cirurgia para o câncer de pulmão $(25,6 \%)$ e uma menor sobrevida específica nos pacientes com pelo menos uma cirurgia para o câncer de pulmão (23,8\%). Houve uma maior sobrevida específica nos pacientes com procedimento radioterápico principal ou secundário $(25,9 \%)$ e uma menor sobrevida específica nos pacientes sem nenhum procedimento radioterápico principal

Tabela 2. Análise de sobrevida global pelo método de Kaplan-Meier dos pacientes com câncer de pulmão tratados no SUS entre 2002 e 2003

\begin{tabular}{|c|c|c|c|}
\hline Variável & $\begin{array}{l}\text { Total } \\
\text { n (\%) }\end{array}$ & $\begin{array}{c}\text { Eventos } \\
\text { n (\%) }\end{array}$ & $\begin{array}{c}\text { Censurados } \\
\text { n (\%) }\end{array}$ \\
\hline \multicolumn{4}{|l|}{ Sexo } \\
\hline Feminino & $3.552(31,8)$ & $3.139(88,4)$ & $413(11,6)$ \\
\hline Masculino & $7.605(68,2)$ & $6.926(91,1)$ & $679(8,9)$ \\
\hline \multicolumn{4}{|l|}{ Idade } \\
\hline $19-49$ & $1.593(14,3)$ & $1.385(86,9)$ & $208(13,1)$ \\
\hline $50-59$ & $2.871(25,7)$ & $2.557(89,1)$ & $314(10,9)$ \\
\hline $60-69$ & $3.807(34,1)$ & $3.481(91,4)$ & $326(8,6)$ \\
\hline $70-100$ & $2.886(25,9)$ & $2.642(91,5)$ & $244(8,5)$ \\
\hline \multicolumn{4}{|l|}{ Região de nascimento } \\
\hline Sudeste & $5.346(47,9)$ & $4.917(92,0)$ & $429(8,0)$ \\
\hline Sul & $2.945(26,4)$ & $2.695(91,5)$ & $250(8,5)$ \\
\hline Centro-Oeste & $564(5,1)$ & $497(88,1)$ & $67(11,9)$ \\
\hline Norte & $350(3,1)$ & $284(81,1)$ & $66(18,9)$ \\
\hline Nordeste & $1.822(16,3)$ & $1.551(85,1)$ & $271(14,9)$ \\
\hline Naturalizados e estrangeiros & $130(1,2)$ & $121(93,1)$ & $9(6,9)$ \\
\hline \multicolumn{4}{|l|}{ Região de residência no início do tratamento } \\
\hline Sudeste & $5.526(49,5)$ & $5.077(91,9)$ & $449(8,1)$ \\
\hline Sul & $3.084(27,6)$ & $2.825(91,6)$ & $259(8,4)$ \\
\hline Centro-Oeste & $592(5,3)$ & $519(87,7)$ & $73(12,3)$ \\
\hline Norte & $362(3,2)$ & $291(80,4)$ & $71(19,6)$ \\
\hline Nordeste & $1.593(14,3)$ & $1.353(84,9)$ & $240(15,1)$ \\
\hline \multicolumn{4}{|l|}{ Estadiamento ao diagnóstico } \\
\hline Doença precoce (estádios 0, I e II) & $1.254(11,2)$ & $1.078(86,0)$ & $176(14,0)$ \\
\hline Doença avançada (estádios III e IV) & $9.903(88,8)$ & $8.987(90,8)$ & $916(9,2)$ \\
\hline \multicolumn{4}{|l|}{ Cirurgia } \\
\hline Nenhuma cirurgia para câncer de pulmão & $10.943(98,1)$ & $9.873(90,2)$ & $1.070(9,8)$ \\
\hline Pelo menos uma cirurgia para câncer de pulmão & $214(1,9)$ & $192(89,7)$ & $22(10,3)$ \\
\hline \multicolumn{4}{|l|}{ Radioterapia } \\
\hline Nenhum procedimento principal ou secundário & $4.080(36,6)$ & $3.702(90,7)$ & $378(9,3)$ \\
\hline Procedimento principal ou secundário & $7.077(63,4)$ & $6.363(89,9)$ & $714(10,1)$ \\
\hline \multicolumn{4}{|l|}{ Quimioterapia } \\
\hline Nenhuma & $3.148(28,2)$ & $2.791(88,7)$ & $357(11,3)$ \\
\hline Procedimento quimioterápico & $8.009(71,8)$ & $7.274(90,8)$ & $735(9,2)$ \\
\hline \multicolumn{4}{|l|}{ Ocorrência de metástase } \\
\hline Não & $5.581(50,0)$ & $4.981(89,2)$ & $600(10,8)$ \\
\hline Sim & $5.576(50,0)$ & $5.084(91,2)$ & $492(8,8)$ \\
\hline \multicolumn{4}{|l|}{ Ocorrência de outro câncer principal } \\
\hline Não teve outro câncer primário & $10.749(96,3)$ & $9.747(90,7)$ & $1.002(9,3)$ \\
\hline Teve outro câncer primário & $408(3,7)$ & $318(77,9)$ & $90(22,1)$ \\
\hline
\end{tabular}

Fonte: Base Nacional em Oncologia

Nota: teste de Log-Rank significativo: $\mathrm{p}<0,001$ 
ou secundário (25,0\%). Houve uma maior sobrevida específica nos pacientes com procedimento quimioterápico $(24,2 \%)$ e uma menor sobrevida específica nos pacientes sem nenhum procedimento quimioterápico (11,3\%). Houve uma maior sobrevida específica nos pacientes sem metástase à distância $(25,7 \%)$ e uma menor sobrevida específica nos pacientes com metástase à distância (25,4\%). Houve uma maior sobrevida específica nos pacientes que tiveram outro câncer principal $(55,1 \%)$ e uma menor sobrevida específica nos pacientes que não tiveram outro câncer principal (24,4\%).
$\mathrm{Na}$ análise de sobrevida com o modelo de Cox, as covariáveis modalidade de tratamento (cirurgia, radioterapia e/ou quimioterapia) foram excluídas por haverem dados insuficientes e a covariável tempo entre diagnóstico e ocorrência de metástase também foi excluída, pois como houve metástase em $50 \%$ dos casos, os outros $50 \%$ não tiveram tempo entre diagnóstico e ocorrência de metástase, portanto houve perda de $50 \%$ dos dados, as demais covariáveis, sexo, idade, região de nascimento, região de residência, estadiamento no momento do diagnóstico, ocorrência de metástase, tempo entre diagnóstico e entrada em tratamento oncológico

Tabela 3. Análise de sobrevida específica pelo método de Kaplan-Meier dos pacientes com câncer de pulmão tratados no SUS entre 2002 e 2003

\begin{tabular}{|c|c|c|c|}
\hline Variável & $\begin{array}{l}\text { Total } \\
\text { n (\%) }\end{array}$ & $\begin{array}{c}\text { Eventos } \\
\text { n (\%) }\end{array}$ & $\begin{array}{c}\text { Censurados } \\
\text { n (\%) }\end{array}$ \\
\hline \multicolumn{4}{|l|}{ Sexo } \\
\hline Feminino & $3.552(31,8)$ & $2.566(72,2)$ & $986(27,8)$ \\
\hline Masculino & $7.605(68,2)$ & $5.738(75,5)$ & $1.867(24,5)$ \\
\hline \multicolumn{4}{|l|}{ Idade } \\
\hline $19-49$ & $1.593(14,3)$ & $1.125(70,6)$ & $468(29,4)$ \\
\hline $50-59$ & $2.871(25,7)$ & $2.140(74,5)$ & $731(25,5)$ \\
\hline $60-69$ & $3.807(34,1)$ & $2.858(75,1)$ & $949(24,9)$ \\
\hline $70-100$ & $2.886(25,9)$ & $2.181(75,6)$ & $705(24,4)$ \\
\hline \multicolumn{4}{|l|}{ Região de nascimento } \\
\hline Sudeste & $5.346(47,9)$ & $4.131(77,3)$ & $1.215(22,7)$ \\
\hline Sul & $2.945(26,4)$ & $2.333(79,2)$ & $612(20,8)$ \\
\hline Centro-Oeste & $564(5,1)$ & $411(72,9)$ & $153(27,1)$ \\
\hline Norte & $350(3,1)$ & $197(56,3)$ & $153(43,7)$ \\
\hline Nordeste & $1.822(16,3)$ & $1.127(61,9)$ & $695(38,1)$ \\
\hline Naturalizados e estrangeiros & $130(1,2)$ & $105(80,8)$ & $25(19,2)$ \\
\hline \multicolumn{4}{|l|}{ Região de residência } \\
\hline Sudeste & $5.526(49,5)$ & $4.271(77,3)$ & $1.255(22,7)$ \\
\hline Sul & $3.084(27,6)$ & $2.446(79,3)$ & $638(20,7)$ \\
\hline Centro-Oeste & $592(5,3)$ & $432(73,0)$ & $160(27,0)$ \\
\hline Norte & $362(3,2)$ & $197(54,4)$ & $165(45,6)$ \\
\hline Nordeste & $1.593(14,3)$ & $958(60,1)$ & $635(39,9)$ \\
\hline \multicolumn{4}{|l|}{ Estadiamento } \\
\hline Doença precoce (estádios 0, I e II) & $1.254(11,2)$ & $876(69,9)$ & $378(30,1)$ \\
\hline Doença avançada (estádios III e IV) & $9.903(88,8)$ & $7.428(75,0)$ & $2.475(25,0)$ \\
\hline \multicolumn{4}{|l|}{ Cirurgia } \\
\hline Nenhuma cirurgia para câncer de pulmão & $10.943(98,1)$ & $8.141(74,4)$ & $2.802(25,6)$ \\
\hline Pelo menos uma cirurgia para câncer de pulmão & $214(1,9)$ & $163(76,2)$ & $51(23,8)$ \\
\hline \multicolumn{4}{|l|}{ Radioterapia } \\
\hline Nenhum procedimento principal ou secundário & $4.080(36,6)$ & $3.062(75,0)$ & $1.018(25,0)$ \\
\hline Procedimento principal ou secundário & $7.077(63,4)$ & $5.242(74,1)$ & $1.835(25,9)$ \\
\hline \multicolumn{4}{|l|}{ Quimioterapia } \\
\hline Nenhum procedimento quimioterápico & $3.148(28,2)$ & $2791(88,7)$ & $357(11,3)$ \\
\hline Procedimento quimioterápico & $8.009(71,8)$ & $6.067(75,8)$ & $1.942(24,2)$ \\
\hline \multicolumn{4}{|l|}{ Ocorrência de metástase } \\
\hline Não & $5.581(50,0)$ & $4.145(74,3)$ & $1.436(25,7)$ \\
\hline Sim & $5.576(50,0)$ & $4.159(74,6)$ & $1.417(25,4)$ \\
\hline \multicolumn{4}{|l|}{ Ocorrência de outro câncer principal } \\
\hline Não teve outro câncer primário & $10.749(96,3)$ & $8.121(75,6)$ & $2.628(24,4)$ \\
\hline Teve outro câncer primário & $408(3,7)$ & $183(44,9)$ & $225(55,1)$ \\
\hline
\end{tabular}

Fonte: Base Nacional em Oncologia

Nota: teste de Log-Rank significativo: $p<0,001$ 
e ocorrência de outro câncer principal apresentaram $\mathrm{p}<0,05$, ou seja, todas foram estatisticamente significantes, tanto na sobrevida global como na sobrevida específica (Tabela 4).

A sobrevida para o câncer de pulmão inferior a 1 ano foi de $45 \%$ e a sobrevida para o câncer de pulmão superior a 5 anos foi de $16 \%$ para os pacientes submetidos à radioterapia e/ou à quimioterapia com ou sem cirurgia associada, para o tratamento do câncer de pulmão no SUS no período de $1^{\circ} \mathrm{de}$ janeiro de 2002 a 31 de dezembro de 2003.

\section{DISCUSSÃO}

$\mathrm{O}$ câncer de pulmão é uma doença que acomete ambos os sexos, porém vê-se neste estudo que o sexo masculino foi o mais acometido, provavelmente pelo maior consumo de tabaco, fato que tende a mudar com o passar do tempo, pois está havendo um aumento no consumo de tabaco entre as mulheres ultimamente, $o$ que deve ser refletido mais a frente, com um aumento na incidência do câncer de pulmão no sexo feminino. Além disso, neste estudo, o câncer de pulmão mostrou ser uma doença com predileção pela

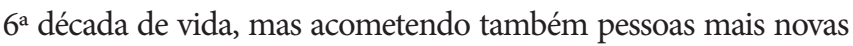
ou mais velhas. Foi detectado mais casos no Sudeste e no Sul, provavelmente pela maior densidade populacional e pela maior rede de serviços de saúde ofertados nessas regiões. A maioria dos pacientes foi diagnosticada em estádios avançados (III e IV) e o óbito por câncer de pulmão foi o desfecho mais comum ${ }^{5,8,9}$.

Quando se analisa o prognóstico do câncer de pulmão relacionado ao sexo, nosso estudo evidenciou uma maior sobrevida específica no sexo feminino. Desde a década de 1980, alguns estudos haviam demonstrado que o sexo feminino poderia ser um fator preditor independente favorável, especialmente em casos de doença precoce, mas ainda não pode ser tirada essa conclusão, sendo necessárias mais pesquisas nesse sentido ${ }^{5,8,10}$.

A idade mostrou-se um fator independente na sobrevida nesta coorte. O melhor prognóstico do câncer de pulmão foi

Tabela 4. Análise de sobrevida global e específica pelo método de Cox dos pacientes com câncer de pulmão tratados no SUS entre 2002 e 2003

\begin{tabular}{|c|c|c|c|c|}
\hline \multirow[b]{2}{*}{ Variáveis } & \multicolumn{2}{|c|}{ Sobrevida Global } & \multicolumn{2}{|c|}{ Sobrevida Específica } \\
\hline & $\begin{array}{l}\text { Hazard ratio } \\
\text { (IC95\%) }\end{array}$ & Valor $\mathbf{p}$ & $\begin{array}{l}\text { Hazard ratio } \\
\text { (IC95\%) }\end{array}$ & Valor $\mathrm{p}$ \\
\hline \multicolumn{5}{|l|}{ Sexo } \\
\hline Feminino & $0,88(0,84-0,91)$ & $<0,001^{\star}$ & $0,87(0,83-0,91)$ & $<0,001^{\star}$ \\
\hline Masculino & 1,00 & & 1,00 & \\
\hline \multicolumn{5}{|l|}{ Grupo de idade } \\
\hline $19-49$ & $0,84(0,79-0,90)$ & $<0,001^{*}$ & $0,84(0,78-0,90)$ & $<0,001^{\star}$ \\
\hline $50-59$ & $0,91(0,86-0,96)$ & $<0,001^{\star}$ & $0,93(0,87-0,98)$ & $0,011^{*}$ \\
\hline $60-69$ & $0,94(0,89-0,98)$ & $0,010^{*}$ & $0,93(0,88-0,98)$ & $0,009^{*}$ \\
\hline $70+$ & 1,00 & & 1,00 & \\
\hline \multicolumn{5}{|l|}{ Região de nascimento } \\
\hline Sudeste & $0,67(0,55-0,82)$ & $<0,001^{*}$ & $0,62(0,50-0,77)$ & $<0,001^{\star}$ \\
\hline Sul & $0,69(0,56-0,84)$ & $<0,001^{\star}$ & $0,65(0,52-0,80)$ & $<0,001^{\star}$ \\
\hline Centro-Oeste & $0,81(0,60-1,11)$ & 0,187 & $0,72(0,52-1,01)$ & 0,058 \\
\hline Norte & $0,76(0,53-1,09)$ & 0,141 & $0,76(0,50-1,14)$ & 0,179 \\
\hline Nordeste & $0,64(0,52-0,79)$ & $<0,001^{\star}$ & $0,59(0,47-0,74)$ & $<0,001^{\star}$ \\
\hline Naturalizados e estrangeiros & 1,00 & & 1,00 & \\
\hline \multicolumn{5}{|l|}{ Região de Residência no Início do tratamento } \\
\hline Sudeste & $1,20(1,06-1,36)$ & $0,004^{*}$ & $1,43(1,25-1,64)$ & $<0,001^{\star}$ \\
\hline Sul & $1,18(0,99-1,42)$ & 0,073 & $1,41(1,16-1,73)$ & $0,001^{\star}$ \\
\hline Centro-Oeste & $0,93(0,72-1,20)$ & 0,579 & $1,13(0,86-1,50)$ & 0,383 \\
\hline Norte & $0,75(0,55-1,03)$ & 0,072 & $0,68(0,47-0,97)$ & $0,036^{*}$ \\
\hline Nordeste & 1,00 & & 1,00 & \\
\hline \multicolumn{5}{|l|}{ Estadiamento ao diagnóstico } \\
\hline Doença precoce & $0,83(0,78-0,89)$ & $<0,001^{*}$ & $0,82(0,76-0,88)$ & $<0,001^{*}$ \\
\hline Doença avançada & 1,00 & & & \\
\hline \multicolumn{5}{|l|}{ Ocorrência de metástase } \\
\hline Não & $0,86(0,83-0,90)$ & $<0,001^{*}$ & $0,88(0,84-0,92)$ & $<0,001^{*}$ \\
\hline Sim & 1,00 & & & \\
\hline $\begin{array}{l}\text { Tempo em meses do diagnóstico à entrada } \\
\text { em tratamento }\end{array}$ & $0,998(0,998-0,998)$ & $<0,001^{*}$ & $0,998(0,998-0,998)$ & $<0,001^{\star}$ \\
\hline \multicolumn{5}{|l|}{ Ocorrência de outro câncer primário } \\
\hline Não & $1,51(1,34-1,69)$ & $<0,001^{*}$ & $2,08(1,79-2,41)$ & $<0,001^{*}$ \\
\hline Sim & 1,00 & & 1,00 & \\
\hline
\end{tabular}

${ }^{*}$ p-valor significativo com erro de $5 \%$

Fonte: Base Nacional em Oncologia 
na faixa etária $<50$ anos e o pior prognóstico do câncer de pulmão foi na faixa etária $\geq 70$ anos $^{5}$.

Em relação à região de nascimento e à região de residência, vê-se que o prognóstico é melhor no Norte, seguido pelo Nordeste, e pior no Sul, seguido pelo Sudeste, excetuando-se os pacientes naturalizados e estrangeiros, o que pode ser explicado pela precária rede de serviços de saúde instalada no Norte e Nordeste, refletindo uma baixa taxa de diagnóstico e tratamento. Além disso, evidencia-se a universalidade ao acesso no SUS, visto que são encontrados pacientes estrangeiros em diagnóstico e tratamento para o câncer de pulmão no Brasil ${ }^{11}$.

Em concordância com a literatura, a comparação entre as curvas de sobrevida para doença precoce (estádios 0, I e II) e doença avançada (estádios III e IV), revelou comportamento mais agressivo e evolução mais rápida da doença em estádios mais avançados ${ }^{5,8}$.

Com relação ao tipo de tratamento, houve um melhor prognóstico nos pacientes submetidos a radioterapia e/ou quimioterapia, porém não foi evidenciado o mesmo para cirurgia. Como este banco de dados é principalmente de APAC e com interface nas AIH, não foram incorporadas as cirurgias isoladas, portanto fica difícil concluir qualquer coisa a respeito das cirurgias para tratamento do câncer de pulmão.

Com relação à metástase à distância houve um melhor prognóstico nos pacientes sem metástase à distância, compatível com a literatura, na qual o pior prognóstico é nos estádios mais avançados ${ }^{5,8}$.

Com relação a outro câncer principal houve um melhor prognóstico nos pacientes que desenvolveram outro câncer principal.

A sobrevida do câncer de pulmão inferior a 1 ano foi de $45 \%$ e a sobrevida do câncer de pulmão superior a 5 anos foi de $16 \%$, não evidenciando diferenças entre países desenvolvidos e países em desenvolvimento, visto que o diagnóstico é tardio e difícil, e a sobrevida reduzida ${ }^{5,8}$.

\section{CONCLUSÃO}

Neste estudo, o câncer de pulmão foi de difícil detecção precoce, tendo sido detectado em maior medida em estádios avançados (III e IV), sendo compatível com a literatura. A maioria dos pacientes evoluiu para óbito relacionado ao câncer. Conclui-se então que o câncer de pulmão é uma doença grave, de difícil diagnóstico e de difícil tratamento, sendo a prevenção a melhor conduta.

O hábito de consumo do tabaco é o maior fator de risco para o câncer de pulmão, e o sexo masculino, apesar de ser o grande consumidor de tabaco e de ter a maior incidência do câncer de pulmão no Brasil, vem apresentando uma tendência de desaceleração na curva de mortalidade por câncer de pulmão, por outro lado, o sexo feminino vem aumentando o consumo de tabaco e vem apresentando uma tendência de aceleração na curva de mortalidade por câncer de pulmão. Conclui-se, pois, que o consumo de tabaco continua ocorrendo em níveis alarmantes e que, mesmo o Brasil tendo uma das legislações mais rigorosas do mundo no combate ao tabaco, com a proibição do consumo de tabaco em lugares fechados, sejam estes públicos ou privados, e a extinção dos fumódromos, apesar de a redução do consumo de tabaco na população em geral ter sido detectada pelo Ministério da Saúde, que relatou uma queda do consumo de tabaco na população em geral entre $2006 \mathrm{e}$ 2010 de 16,2\% para 15,1\%, com redução mais expressiva no sexo masculino, ainda assim faz-se necessário medidas mais duras para erradicarmos esta pandemia que é o consumo de tabaco.

Com relação ao asbesto, o Brasil é um dos maiores países produtores, consumidores e exportadores de amianto no mundo, e ainda nos faltam leis e uma maior conscientização do grave problema de saúde pública ocasionado por essa prática comercial que deveria ser banida do Brasil e do mundo.

Ao mesmo tempo em que devemos melhorar e expandir a rede SUS nas regiões Norte, Nordeste e Centro-Oeste, onde o diagnóstico do câncer de pulmão, provavelmente na grande maioria dos casos, deixou de ser feito, também não podemos nos esquecer da rede SUS nas regiões Sul e Sudeste, pois apesar de mais bem implantada ainda há muitas deficiências a serem sanadas.

Com relação aos Recursos Humanos no SUS, nós devemos implementar uma política de estado para fixar os médicos no interior, talvez com a construção de um Plano de Carreira, Cargos e Salários, nos moldes do que ocorre no Ministério Público e no Judiciário, nós também devemos fomentar as Residências Médicas de Medicina de Família e Comunidade para capacitar os médicos nas Unidades Básicas de Saúde (UBS), que são a porta de entrada do SUS, visto que o diagnóstico e o tratamento do câncer no SUS é feito tardiamente, em parte pela má formação do profissional da atenção básica, em parte pela dificuldade de acesso aos serviços especializados, em parte pela superlotação na rede e em parte pela carência de equipamentos médicos diagnósticos e terapêuticos distribuídos de forma não equitativa pela rede SUS.

Combinando prevenção, diagnóstico e tratamento mais eficazes contra o câncer de pulmão, quem sabe não vencemos esta guerra que por ora estamos perdendo.

\section{AGRADECIMENTOS}

Os autores agradecem ao Grupo de Pesquisa em Economia da Saúde (GPES), ao Centro Nacional de Desenvolvimento Científico e Tecnológico (CNPq) (Processos 409729/2006-0 e 479033/2010-2) e à Fundação de Amparo à Pesquisa do Estado de Minas Gerais (FAPEMIG) (Processo EDT 3284/06 e Processo CDS - PPM-00098-11). 


\section{REFERÊNCIAS}

1. Brasil. Ministério da Saúde (BR) [Internet]. Brasília (DF). [cited 2012 Jun 06] Available from http://www.saude.gov.br/

2. Instituto Nacional do Câncer (INCA) [Internet]. Brasília (DF). [cited 2012 Jun 06] Available from http://www.inca.gov.br/

3. World Health Organization (WHO). [Internet]. [cited 2012 Jun 06] Available from http://www.who.int/en/

4. Pollock RE. Manual de Oncologia Clínica da UICC. São Paulo: Fundação Oncocentro de São Paulo; 2006. 919 p.

5. Jamnik S, Santoro IL, Borges EL, Uehara S, Silva VV. Estudo Comparativo de Fatores Prognósticos em Portadores de Carcinoma Não-Pequenas Células de Pulmão: Sobrevida Superior a Cinco Anos e Inferior a Um Ano. Rev Bras Cancerol. 2009;55(1):5-10.

6. Butler CA, Darragh KM, Currie GP, Anderson WJA. Variation in lung cancer survival rates between countries: Do differences in data reporting contribute? Respir Med. 2006;100:1642-6.

7. Queiroz OV, Augusto AG Jr, Machado CJ, Andrade EIG, Wagner M Jr, Acúrcio FA, et al. A construção da base de dados nacional em terapia renal substitutiva (TRS) centrada no indivíduo: aplicação do método de linkage determinístico-probabilístico. Epidemiol Serv Saúde. 2009;18(2):107-20.

8. Novaes FT, Cataneo DC, Raul LR Jr, Defaveri J, Michelin OC, Cataneo AJM. Câncer de pulmão: histologia, estádio, tratamento e sobrevida. J Bras Pneumol. 2008;34(8):595-600.

9. Siegel R, Naishadham D, Jemal. A. Cancer Statistics, 2012. Cancer J Clin. 2012;62(1):10-29.

10. Abreu CM, Chatkin JM, Fritscher CC, Wagner MB, Pinto JAF. Sobrevida de longo prazo em carcinoma brônquico após tratamento cirúrgico: sexo é fator prognóstico? J Bras Pneumol. 2004;30(1):2-8.

11. Shugarman LR, Sorbero MES, Tian H, Jain AK, Ashwood JS. An Exploration of Urban and Rural Differences in Lung Cancer Survival Among Medicare Beneficiaries. Am J Public Health. 2008;98:1280-7.

Recebido em: 16/03/2013 Aprovado em: 12/06/2013 